\title{
A multifunctional molecularly imprinted polymer-based biosensor for direct detection of doxycycline in food samples
}

\author{
Ashley, Jon; Feng, Xiaotong; Sun, Yi
}

Published in:

Talanta

Link to article, DOI:

10.1016/j.talanta.2018.01.056

Publication date:

2018

Document Version

Peer reviewed version

Link back to DTU Orbit

Citation (APA):

Ashley, J., Feng, X., \& Sun, Y. (2018). A multifunctional molecularly imprinted polymer-based biosensor for direct detection of doxycycline in food samples. Talanta, 182, 49-54.

https://doi.org/10.1016/j.talanta.2018.01.056

\section{General rights}

Copyright and moral rights for the publications made accessible in the public portal are retained by the authors and/or other copyright owners and it is a condition of accessing publications that users recognise and abide by the legal requirements associated with these rights.

- Users may download and print one copy of any publication from the public portal for the purpose of private study or research.

- You may not further distribute the material or use it for any profit-making activity or commercial gain

- You may freely distribute the URL identifying the publication in the public portal

If you believe that this document breaches copyright please contact us providing details, and we will remove access to the work immediately and investigate your claim 


\title{
A Multifunctional Molecularly Imprinted Polymer-Based Biosensor for Direct Detection of Doxycycline in Food Samples
}

Jon Ashley, XiaoTong Feng, and Yi Sun*

Department of Micro- and Nanotechnology, Technical University of Denmark, Ørsteds Plads, DK-2800 Kgs, Lyngby, Denmark.

*Corresponding author. Email: sun.yi@nanotech.dtu.dk

\begin{abstract}
In this study, we developed a new type of multifunctional molecularly imprinted polymer (MIP) composite as an all-in-one biosensor for the low-cost, rapid and sensitive detection of doxycycline in pig plasma. The MIP composite consisted of a magnetic core for ease of manipulation, and a shell of fluorescent MIPs for selective recognition of doxycycline. By simply incorporating a small amount of fluorescent monomer (fluorescein-O-acrylate), the fluorescent MIP layer was successfully grafted onto the magnetic core via a surface imprinting technique. The resultant MIP composites showed significant doxycyclinedependent fluorescence quenching in an aqueous environment. Good linearity ranging from 0.2 to $6 \mu \mathrm{M}$ was achieved, and the limit of detection was determined to be $117 \mathrm{nM}$. The biosensor also showed good selectivity towards doxycycline when compared to other common antibiotic residues. The multifunctional MIP composites were used to directly extract doxycycline from spiked pig plasma samples and quantify the antibiotics based on the quenched fluorescence signals. Recoveries of doxycycline were found in the range of 88$107 \%$.
\end{abstract}

\section{Keywords}

Molecularly Imprinted Polymers; Doxycycline; Fluorescence; Multifunctional composite; Pig plasma; Antibiotics 


\section{Introduction}

The overuse of antibiotics is of considerable concern to public health, as these drugs can possibly enter the food chain. These residues can then accumulate in the body to cause adverse health effects as well as increasing the likelihood of antibiotic resistance. Doxycycline, which is one of a broad class of tetracycline antibiotics, has been found to be overprescribed by veterinary professionals to effectively control bacterial pneumonia in pigs and other livestock which has contributed to the bacteria resistance [1]. Screening of doxycycline is compulsory for a number of food matrices. According to the EU commission regulation No 37/2010, the maximum residue limits (MRLs) of doxycycline is $100 \mu \mathrm{g} / \mathrm{kg}$ in muscle, $300 \mu \mathrm{g} / \mathrm{kg}$ in skin, fat and liver, and $600 \mu \mathrm{g} / \mathrm{kg}$ in Kidney [2].

As such, there is a need for the development of rapid, sensitive and low cost analytical methods to accurately detect doxycycline and other tetracycline based antibiotics within different food matrices. Current analytical methods can be split into two general groups, namely confirmatory and screening [3]. Confirmatory methods such as high performance liquid chromatography (HPLC) with UV detection [4], liquid chromatography-mass spectrometry (LC-MS) [5], and capillary electrophoresis (CE) [6,7], have demonstrated high sensitivity towards doxycycline with a high degree of accuracy and precision. However these techniques are often time-consuming, require expensive instrumentation as well as trained personnel to operate them.

Alternatively, screening based methods such as bioassays and biosensors have allowed for the semi-quantitative analysis of drug residues [8-10]. In particular, a number of biosensors for the detection of tetracycline based antibiotic residues in food products have been reported in the literature [11]. They have the potential to be used before confirmatory methods and 
have shown great potential for antibiotic analysis at the point-of-need due to their high throughput analysis, rapid analysis times and miniaturization [12-14]. However, most of the assays rely on bioreceptors such as antibodies and enzymes for specific recognition. In spite of the high specificity and affinity, these bioreceptors are expensive and inherently unstable due to their biological origin.

Recently, molecularly imprinted polymers (MIPs), the synthetic materials with recognition sites that can specifically bind to target molecules, have attracted immense attention. MIPs targeting tetracycline antibiotics have been synthesized by radical polymerization or precipitation polymerization for use in biosensing platforms or as sorbents for solid phase extraction $[15,16]$. Compared to conventional bioreceptors, MIPs offer apparent advantages including higher robustness and stability under a wide range of conditions, ease of synthesis and low production costs. More interestingly, different active molecules can be incorporated during synthesis, so that multifunctional MIP composites can be formed [17,18]. MIPs have been grafted onto the surface of magnetic nanoparticles, resulting in magnetic MIPs which possess both magnetically susceptible characteristics and good selectivity for the target molecules [19-21]. Fluorescent molecules such as fluorescent dyes, quantum dots or carbon dots have also been integrated into MIPs, which effectively turns the MIP composites into fluorescence-based sensors $[22,23]$. Several reports have even demonstrated the combined use of magnetic nanoparticles and fluorophores with MIPs [24,25]. However, there are still technical challenges for synthesizing such multifunctional MIPs. For instance, many examples thus far have used cadmium-based quantum dots which are highly toxic [26]. In addition, the incorporation of fluorescence dyes or quantum dots into the MIP layer might lead to interference in MIP binding. Some work tried to avoid the problem by incorporating quantum dots into a separate silica layer, but this could in turn result in decrease in quenching ability due to the distance between the binding site and fluorescent molecules [22]. 
In this study, we developed a new type of multifunctional MIP composite for the recognition and detection of doxycycline. The MIP composite was synthesized by a core-shell imprinting technique, and consisted of a magnetic core and a shell of fluorescent MIP (FMIP) (Fig. 1). Instead of incorporating quantum dots or fluorescent dyes in MIPs, we developed a facile polymerization method to produce FMIPs by introducing fluorescein-O-acrylate monomer into the molecular imprinting formulation. With a set of combined properties, the new MIP composites were used as a biosensor to directly detect doxycycline in pork plasma samples. Attributed to the magnetic core, the composite could be easily separated and collected using an external magnetic field. The high selectivity of MIPs ensured specific recognition of doxycycline. Upon binding, a fluorescence quenching effect was induced via the interaction between the fluorescein-O-acrylate and the analyte, thereby providing a convenient methodology for optical detection. The use of a fluorescent monomer greatly simplified the synthesis procedures, and the sensitivity was similar or higher when compared to previous methods. The MIP composite-based biosensor was demonstrated to be rapid, simple, selective, and applicable to raw samples. To the best of our knowledge, this is the first reported example of a multifunctional MIP composite for the detection of doxycycline in plasma samples.

\section{Experimental}

\subsection{Materials}

Ferric chloride $\mathrm{FeCl}_{3} \cdot 6 \mathrm{H}_{2} \mathrm{O}$, ethylene glycol, sodium acetate, polyethylene glycol (PEG, MW 2000), tetraethoxysilane (TEOS), 35\% ammonium hydroxide, methacryloxypropyltrimethoxysilane (MPA), doxycycline (DO), cloxacillin (CA), spiramycin (SP), gentamycin (GE), tetracycline (TC), amoxicillin (AC), ethylene glycol dimethacrylate (EDGMA), methacrylic acid (MAA) and azo(bis)isobutyronitrile (AIBN), 
fluorescein-o-acrylate and porcine plasma were purchased from Sigma Aldrich. Inhibitors were removed from all monomers prior to polymerization using pre-packed columns from Sigma Aldrich. All solvents were analytical grade and used without further purification.

\subsection{Synthesis of $\mathrm{FeO}_{\mathrm{x}} @ \mathrm{SiO}_{2}-\mathrm{FMIPs}$}

The synthesis procedures are illustrated in Fig. 1. The iron oxide $\left(\mathrm{FeO}_{\mathrm{x}}\right)$ nanoparticles were synthesized using the thermal solvent method. $\mathrm{FeCl}_{3} \cdot 6 \mathrm{H}_{2} \mathrm{O}$ (1.36 g) was dissolved in ethylene glycol (40 ml). Anhydrous sodium acetate (3.6 g) and PEG (MW 2000, 0.2 g) were added sequentially. The mixture was stirred for 30 mins to form a homogenous orange suspension. The solution was then degassed under vacuum for 30 mins, transferred to a 100 $\mathrm{ml}$ autoclave reactor and heated in a furnace oven at $200{ }^{\circ} \mathrm{C}$ for 10 hrs. The resultant black precipitate was washed several times with ethanol and dried under vacuum for 6 hrs.

$0.5 \mathrm{~g}$ of the resultant $\mathrm{FeO}_{\mathrm{x}}$ microspheres were re-suspended in ethanol $(60 \mathrm{ml})$ and water (12 ml). The black solution was then sonicated using a probe sonicator for at least 30 mins followed by the addition of 35\% ammonia hydroxide (2 ml) and TEOS (2 ml). The solution was allowed to react under pulse sonication for 4 hrs before transferring to an orbital shaker for 12 hrs. The resultant FeOx@SiO2 microspheres were washed several times with water until the $\mathrm{pH}$ had returned to neutral. This was followed by washing with ethanol several times and dried under vacuum for 12 hrs.

The resultant $\mathrm{FeO}_{\mathrm{x}} @ \mathrm{SiO}_{2}(0.4 \mathrm{~g})$ were mixed with 4\% (v:v) MPA: anhydrous toluene. The solution was sonicated for 15 mins and then heated to $60{ }^{\circ} \mathrm{C}$ with overhead stirring under nitrogen overnight. The resultant $\mathrm{FeO}_{\mathrm{x}} @ \mathrm{SiO}_{2}-\mathrm{MPA}$ microspheres were washed several times using ethanol and dried for 10 hrs under vacuum. 
The synthesis of the MIP layer is based on a previous report with some modifications [27]. A solution containing MAA (4 mmol), EDGMA (8 mmol), doxycycline (1 mmol), and fluorescein-o-acrylate $(0.1 \mathrm{mM})$ in methanol $(60 \mathrm{ml})$ was prepared and degassed by bubbling nitrogen for 30 mins. The solution was added to a three-way round bottom flask containing 0.2 g of $\mathrm{FeO}_{\mathrm{x}} @ \mathrm{SiO}_{2}-\mathrm{MPA}$, sonicated for 15 mins and stirred for one hr at room temperature. The polymerization reaction was initiated by adding AIBN (40 mg) and the mixture was heated to $60^{\circ} \mathrm{C}$ for 16 hrs. The resultant $\mathrm{FeO}_{\mathrm{x}} @ \mathrm{SiO}_{2}-\mathrm{FMIPs}$ were transferred to a soxhlet extractor and continually washed using 9:1 (v:v) methanol:acetic acid over a $24 \mathrm{hr}$ period to remove the template. The removal of the template was then confirmed by checking the fluorescence of the microspheres. FeOx@SiOz-FMIPs were finally washed with methanol several times to remove residual acetic acid, and dried under vacuum. The non-imprinted polymers (NIPs) were prepared in the same manner as for the MIPs but in the absence of the template.

\subsection{Instrumentation}

All scanning electron microscopy (SEM) images were taken using a Quanta FEG SEM (FEI, Oregen USA). All transmission electron microscopy (TEM) images were taken using a Tecnai T20 G2 (FEI, Oregen USA) transmission electron microscope. IR spectra were taken using a Spectrum 100 (Perkin Elmer, MA, USA). XPS spectra were measured using a Kalpha XPS (Thermo Fisher Scientific, MA, USA). All fluorescence-based quenching experiments were performed on a Tecan Spark plate reader (TECAN, Switzerland). All hydrodynamic size measurements were performed on a Brookhaven ZetaPAL DLS.

\subsection{Measuring the fluorescent properties of $\mathrm{FeO}_{\mathrm{x}} @ \mathrm{SiO}_{2}-\mathrm{FMIPs}$}

The fluorescence properties of $\mathrm{FeO}_{\mathbf{x}} @ \mathrm{SiO}_{2}-\mathrm{FMIPs}$ were imaged on a fluorescent microscope by drying an aliquot on a glass slide. For the development of the fluorescence quenching 
assays, all measurements were performed on a plate reader equipped with fluorescence detection in a 96-well plate format. All fluorescence experiments were performed in triplicate. A solution of $\mathrm{FeO}_{\mathrm{x}} @ \mathrm{SiO}_{2}-\mathrm{FMIPs}\left(1 \mathrm{mg} \mathrm{ml}^{-1}\right)$ was prepared in 10 mM PBS. After being mixed and sonicated for 15 mins, $100 \mu \mathrm{l}$ of the solution was added to each well. Next, $100 \mu \mathrm{l}$ of doxycycline in PBS with concentrations from 0.2 to $300 \mu \mathrm{M}$ were added. The resultant samples were incubated for $1 \mathrm{hr}$ before measurement. The fluorescence excitation wavelength was set at $470 \mathrm{~nm}$, and the emission was measured at $520 \mathrm{~nm}$ for all fluorescence experiments. The quenching of fluorescence signal was determined by comparing the fluorescence signals to the blank sample (containing just the buffer and the MIPs).

The selectivity of $\mathrm{FeO}_{\mathrm{x}} @ \mathrm{SiO}_{2}-\mathrm{FMIPs}$ was determined by incubating $\mathrm{FeO}_{\mathrm{x}} @ \mathrm{SiO}_{2}-\mathrm{FMIPs}$ and $\mathrm{FeO}_{\mathbf{x}} @ \mathrm{SiO}_{2}$-FNIPs with $6 \mu \mathrm{M}$ of doxycycline, cloxacillin, spiramycin and gentamycin, amoxicillin and tetracycline at room temperature respectively. The response of each antibiotic was determined from the extent of fluorescence quenching after incubation.

\subsection{Real Sample analysis}

The ability of FeOx@SiO2-FMIPs to detect doxycycline in real samples was assessed. $100 \mu \mathrm{l}$ of pig plasma samples spiked with doxycycline at different concentrations (0-6 $\mu \mathrm{M})$ were mixed with 1 mg ml ${ }^{-1}$ of $\mathrm{FeO}_{\mathrm{x}} @ \mathrm{SiO}_{2}-\mathrm{FMIPs}(100 \mu \mathrm{l})$ and incubated for one hr. FeO $@ \mathrm{SiO}_{2}-$ FMIPs were then separated from the plasma samples by magnet and transferred to a well plate. The antibiotic concentration was calculated by fitting the quenched signal to the calibration plot. The sample recovery was determined by comparing the measured concentration to the known spiked concentration.

\section{Results and Discussion}

\subsection{Characterization of $\mathrm{FeO}_{\mathrm{x}} @ \mathrm{SiO}_{2}$-FMIPs}


From the several batches of $\mathrm{FeO}_{\mathrm{x}} @ \mathrm{SiO}_{2}-\mathrm{FMIPs}$ and corresponding $\mathrm{FeO}_{\mathrm{x}} @ \mathrm{SiO}_{2}-\mathrm{FNIPs}$ synthesized, MAA and fluorescein-O-acrylate were used as functional monomers to form a mixture of hydrogen bonding and electrostatic interactions with the amide and hydroxyl groups on the template. These interactions allowed the analyte to be in close proximity to the fluorophore, so that a quenching effect could be induced. The concentration of the fluorescein-o-acrylate was optimised to give the maximum fluorescence intensity as shown in Fig S1. As the concentration of fluorescein-o-acrylate used in the co-polymerization was decreased, the fluorescence intensity after the removal of the template also decreased with $100 \mu \mathrm{M}$ showing the highest intensity. Precipitated monomers and template were observed in pre-polymerization mixtures containing $>100 \mu \mathrm{M}$ acrylate-o-fluorescein. The composition of the FMIP layer was optimised (Table S1), during the co-polymerization to ensure that there was high enough specific quenching upon analyte binding, while at the same time minimizing the formation of the bulk polymer by-product. This was achieved by having a sufficient amount of $\mathrm{FeO}_{\mathrm{x}} @ \mathrm{SiO}_{2}-\mathrm{MPA}$ and reducing the amount of crosslinker to ensure that the majority of polymerization occurred on the surface of the nanoparticle. It is also worth noting that the $\mathrm{FeO}_{\mathrm{x}} @ \mathrm{SiO}_{2}-\mathrm{MPA}$ could act as a crosslinker itself due to the large number of acrylate groups found on the surface. The optimum crosslinker to template ratio was 8:1, while the optimum ratio of monomer to template was 4:1. A high molar amount of template also ensured that there were a high enough number of binding sites on the surface of the polymer.

The morphologies of $\mathrm{FeO}_{\mathrm{x}}, \mathrm{FeO}_{\mathrm{x}} @ \mathrm{SiO}_{2}$, and $\mathrm{FeO}_{\mathbf{x}} @ \mathrm{SiO}_{2}-\mathrm{FMIPs}$ microspheres were determined by SEM and TEM imaging. TEM images of $\mathrm{FeO}_{\mathrm{x}}$ and $\mathrm{FeO}_{\mathrm{x}} @ \mathrm{SiO}_{2}$ (Fig. 2A -B) confirmed the addition of a silica shell layer surrounding the magnetic microspheres. The MIP and corresponding NIP layers were also observed upon polymerization (Fig. 2C-D). The TEM images demonstrated the successive additions of layers onto the magnetic core. 
The corresponding SEM images (Fig. S2) showed spherical particles with a diameter of about $207 \mathrm{~nm}$ for $\mathrm{FeO}_{\mathrm{x}}$ while the size increased to 461nm for $\mathrm{FeO}_{\mathbf{x}} @ \mathrm{SiO}_{2}$. The total diameter of FeOx@SiO2-FMIPs was estimated to be about 510 nm. Each $\mathrm{FeO}_{\mathrm{x}}$ microsphere core was made up of smaller nanoparticles of about $10 \mathrm{~nm}$ in diameter. FeOx, FeOx@SiO2, and $\mathrm{FeO}_{\mathbf{x}} @ \mathrm{SiO}_{2}-\mathrm{FMIPs}$ were further characterized using IR and XPS. The IR spectra of $\mathrm{FeO}_{\mathrm{x}}$, FeO ${ }_{x} @ S_{2}, \quad F_{2} O_{x} @ S_{2} O_{2}-F M I P s$ and $\mathrm{FeO}_{x} @ S^{2} O_{2}-F N I P s$ (Fig. 3) showed the apparent changes in the morphology of the microsphere surface after successive modification steps.

Distinct IR peaks at about 953 and $1079 \mathrm{~cm}^{-1}$ corresponding to Si-O-H stretching vibration and Si-O-Si asymmetric stretching vibrations were observed respectively. The peak observed at $807 \mathrm{~cm}^{-1}$ corresponded to Si-O-Si symmetric stretching vibration. No changes were observed between the IR spectra of $\mathrm{FeO}_{\mathrm{x}} @ \mathrm{SiO}_{2}, \mathrm{FeO}_{\mathrm{x}} @ \mathrm{SiO}_{2}-\mathrm{MPA}$ due to the fact that the MPA formed a monolayer which could not be observed using the IR ATR setup. However, a peak at $1732 \mathrm{~cm}^{-1}$ corresponding to $\mathrm{C}=\mathrm{O}$ confirmed the presence of the MIP layer and the corresponding NIP layer. The characterisation of silane monolayer MPA onto FeOx@SiO2 was confirmed by XPS and confirmed by an increase in the carbon S1 peak suggesting that the MPA was successfully grafted onto the $\mathrm{SiO}_{2}$ layer (Fig. S3).

\subsection{Fluorescence Properties of $\mathrm{FeO}_{\mathrm{x}} @ \mathrm{SiO}_{2}-\mathrm{FMIPs}$}

The FeOx@SiO2-FMIPs were imaged by a fluorescence microscope. As shown in Fig. 4, the resultant $\mathrm{FeO}_{\mathrm{x}} @ \mathrm{SiO}_{2}-\mathrm{FMIPs}$ displayed strong fluorescence signals, suggesting that both an adequate amount of monomer had been incorporated into the MIP, and that the washing step was sufficient to remove most of the template from the MIP.

Fluorescence quenching experiments were performed to determine the degree of binding of doxycycline to the MIP. Stock solutions containing $1 \mathrm{mg} \mathrm{ml}^{-1}$ of MIPs and corresponding NIPs were pipetted into microplate wells followed by the addition of doxycycline at different 
concentrations. The MIPs displayed high fluorescence signal in PBS in the absence of the template (Fig. 5A). As the MIPs were titrated against an increasing concentration of doxycycline, the fluorescence signal decreased to a point of saturation. The corresponding NIP (Fig. 5B) showed a much lower decrease in fluorescence signal as concentration of doxycycline was increased, suggesting that the binding of doxycycline to the MIPs enhanced the quenching effect. The quenched fluorescence was plotted using the Stern-Volmer equation as follows.

$\left(I_{o} / I\right)-1=K_{S V} \times C_{c}$

where $I_{o}$ and $I$ are the initial fluorescence and quenched fluorescence signal respectively. $K_{S V}$ is the quenching constant for doxycycline and $C_{c}$ is the analyte concentration. Linear SternVolmer relationships were observed for the MIPs (Fig. 6A), where the $K_{s v}$ was $0.03627 \mathrm{M}^{-1}$ $\left(r^{2}=0.9952\right)$, with a saturation of the signal at higher concentrations (Fig.6B). The corresponding NIPs also showed a linear Stern-Volmer relationship (Fig. 6C), where the $K_{s v}$ was $0.008223 \mathrm{M}^{-1}\left(\mathrm{r}^{2}=0.9920\right)$ and saturation of the signal at higher concentrations (Fig. 6D). The non-linear relationship observed at higher concentrations was due to the saturation of the binding sites on the surface of the FMIPs with doxycycline. The FMIPs displayed a 6-fold increase in quenched signal when compared against the corresponding NIPs. The detection limit was determined to be $117 \mathrm{nM}(52 \mu \mathrm{g} / \mathrm{kg})$ and the linear region was between $0.2 \mu \mathrm{M}$ and $6 \mu \mathrm{M}$. Compared to other methods reported for doxycycline detection, such as fluorescence (LOD: $110 \mathrm{nM} ; 49 \mu \mathrm{g} / \mathrm{kg}$ ) [28], electrochemical (LOD: $43.5 \mu \mathrm{M} ; 19.3 \mathrm{mg} / \mathrm{kg}$ ) [29] and spectrophotometric (LOD: $710 \mathrm{nM} ; 315 \mu \mathrm{g} / \mathrm{kg}$ ) [30] sensors, our multifunctional FMIPs demonstrated comparable or higher sensitivity.

The selectivity of $\mathrm{FeO}_{\mathrm{x}} @ \mathrm{SiO}_{2}$-FMIPs against other antibiotics was assessed by incubating each antibiotic with $1 \mathrm{mg} \mathrm{ml}^{-1}$ of $\mathrm{FeO}_{\mathrm{x}} @ \mathrm{SiO}_{2}-\mathrm{FMIPs}$ at the highest concentration found in 
the linear-range of the Stern-Volmer calibration plot which was $6 \mu \mathrm{M}$. The FeOx@SiO $2-$ FMIPs demonstrated a 25-fold increase in quenching towards doxycycline compared to gentamycin, a 17-fold increase in quenching when compared to spiramycin and a 7 to 8-fold increase compared to cloxacillin and amoxicillin and tetracycline (Fig. 7). The doxycycline FMIPs also showed a significant increase in quenching when compared to the corresponding FNIPs for all the antibiotics tested. These results confirmed that the developed multifunctional FMIPs had high selectivity towards doxycycline.

To test the effect of photo bleaching, the fluorescence signal was continually monitored over a 40 minute period with the fluorescence signal measured every 30 seconds (Fig. S4). There was no observed decrease in signal during the experiment, suggesting that the fluorescent monomer did not undergo significant photo-bleaching in PBS buffer for the entire experiment, and the $\mathrm{FeO}_{\mathrm{x}} @ \mathrm{SiO}_{2}-\mathrm{FMIPs}$ could be reused provided that adequate removal of the template can be achieved.

\subsection{Real Sample Analysis}

To test the feasibility of the biosensor to detect doxycycline in real sample analysis, spiked samples containing different known concentrations of analyte were prepared and mixed with the $\mathrm{FeO}_{\mathrm{x}} @ \mathrm{SiO}_{2}-\mathrm{FMIPs}$. The $\mathrm{FeO}_{\mathrm{x}} @ \mathrm{SiO}_{2}-\mathrm{FMIP}$ microspheres were then separated with a magnet, re-suspended in PBS buffer and transferred to a plate well. Attributed to the magnetic property of the MIP composite, doxycycline was effectively extracted from the matrix. By using the blank sample containing MIPs and non-spiked serum as negative control, we could effectively remove the sample matrix effects, allowing the assay to be carried out without additional washing or sample purification steps. Fluorescence quenching was determined according to the Stern-Volmer equation and interpolated against the calibration plot. As shown in Table 1, recoveries of doxycycline from spiked pig plasma 
samples ranged from 88-107 \% with RSD values ranging from 1.15 to $8.1 \%$. These results suggested that the fluorescent $\mathrm{FeO}_{\mathrm{x}} @ \mathrm{SiO}_{2}-\mathrm{FMIPs}$ were capable of determining doxycycline directly in serum with minimal sample pre-treatment. The simple operation procedures and easy manipulation of the MIP composites make it possible to utilize the assay for online or atsite screening of antibiotic residues in food samples.

\section{Conclusion}

In this study, we demonstrated the use of fluorescent $\mathrm{FeO}_{\mathrm{x}} @ \mathrm{SiO}_{2}-\mathrm{FMIPs}$ as an all-in-one biosensor for the detection of doxycycline in pig plasma. Core-shell MIP composites were

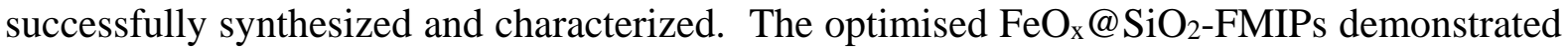
both sensitive and selective quenching in the presence of doxycycline, and the magnetic property of the microspheres enabled the assay to be performed in situ. The assay shows great potential as a low-cost, rapid, high-throughput analysis tool for point-of-need applications.

\section{Acknowledgement}

This work was financially supported by the Villum Fonden, Denmark, project No. 13153.

\section{References}

[1] X. Li, S. Xie, Y. Pan, W. Qu, Y. Tao, D. Chen, L. Huang, Z. Liu, Y. Wang, Z. Yuan, Preparation, characterization and pharmacokinetics of doxycycline hydrochloride and florfenicol polyvinylpyrroliddone microparticle entrapped with hydroxypropyl- $\beta$ cyclodextrin inclusion complexes suspension, Colloids Surfaces B Biointerfaces. 141 (2016) 634-642. doi:10.1016/j.colsurfb.2016.02.027.

[2] The European Commission, Commission Regulation (EU) No 37/2010 of 22 December 2009 on pharmacologically active substances and their classification regarding 
maximum residue limits in foodstuffs of animal origin, Off. J. Eur. Union. L15 (2010) 1-72. doi:2004R0726 - v.7 of 05.06.2013.

[3] C. Cháfer-Pericás, Á. Maquieira, R. Puchades, Fast screening methods to detect antibiotic residues in food samples, TrAC Trends Anal. Chem. 29 (2010) 1038-1049. doi:10.1016/j.trac.2010.06.004.

[4] A.R. Shalaby, N.A. Salama, S.H. Abou-Raya, W.H. Emam, F.M. Mehaya, Validation of HPLC method for determination of tetracycline residues in chicken meat and liver, Food Chem. 124 (2011) 1660-1666. doi:10.1016/j.foodchem.2010.07.048.

[5] A. Gajda, A. Posyniak, G. Tomczyk, LC-MS/MS analysis of doxycycline residues in chicken tissues after oral administration, Bull. Vet. Inst. Pulawy. 58 (2014) 573-579. doi:10.2478/bvip-2014-0089.

[6] A.M. García-Campaña, L. Gámiz-Gracia, F.J. Lara, M. del Olmo Iruela, C. CrucesBlanco, Applications of capillary electrophoresis to the determination of antibiotics in food and environmental samples, Anal. Bioanal. Chem. 395 (2009) 967-986. doi:10.1007/s00216-009-2867-9.

[7] G. Mu, H. Liu, L. Xu, L. Tian, F. Luan, Matrix Solid-Phase Dispersion Extraction and Capillary Electrophoresis Determination of Tetracycline Residues in Milk, Food Anal. Methods. 5 (2012) 148-153. doi:10.1007/s12161-011-9225-1.

[8] J. Adrian, F. Fernández, F. Sánchez-Baeza, M.P. Marco, Preparation of antibodies and development of an enzyme-linked immunosorbent assay (ELISA) for the determination of doxycycline antibiotic in milk samples, J. Agric. Food Chem. 60 (2012) 3837-3846. doi:10.1021/jf2053355.

[9] K. Reder-Christ, G. Bendas, Biosensor Applications in the Field of Antibiotic 
Research—A Review of Recent Developments, Sensors (Basel). 11 (2011) 9450_ 9466. doi:10.3390/s111009450.

[10] M.G. Pikkemaat, S.O. v Dijk, J. Schouten, M. Rapallini, L. Kortenhoeven, H.J. van Egmond, Nouws antibiotic test: Validation of a post-screening method for antibiotic residues in kidney, Food Control. 20 (2009) 771-777. doi:10.1016/j.foodcont.2008.10.001.

[11] A. Wang, Y. Bai, H. Gao, S. Wang, A tetracycline-selective fluorescent biosensor using anthranilic acid immobilized on a glutaraldehyde-coated eggshell membrane, Anal. Methods. 7 (2015) 6842-6847. doi:10.1039/C5AY01047K.

[12] N. Karaseva, T. Ermolaeva, B. Mizaikoff, Piezoelectric sensors using molecularly imprinted nanospheres for the detection of antibiotics, Sensors Actuators, B Chem. 225 (2016) 199-208. doi:10.1016/j.snb.2015.11.045.

[13] S. Kesavan, D.R. Kumar, Y.R. Lee, J.J. Shim, Determination of tetracycline in the presence of major interference in human urine samples using polymelamine/electrochemically reduced graphene oxide modified electrode, Sensors Actuators, B Chem. 241 (2017) 455-465. doi:10.1016/j.snb.2016.10.091.

[14] L. Lan, Y. Yao, J. Ping, Y. Ying, Recent advances in nanomaterial-based biosensors for antibiotics detection, Biosens. Bioelectron. 91 (2017) 504-514. doi:10.1016/j.bios.2017.01.007.

[15] L.-Q. Wang, F.-Y. Lin, L.-P. Yu, A molecularly imprinted photonic polymer sensor with high selectivity for tetracyclines analysis in food, Analyst. 137 (2012) 3502. doi:10.1039/c2an35460h.

[16] T. Jing, Y. Wang, Q. Dai, H. Xia, J. Niu, Q. Hao, S. Mei, Y. Zhou, Preparation of 
mixed-templates molecularly imprinted polymers and investigation of the recognition ability for tetracycline antibiotics, Biosens. Bioelectron. 25 (2010) 2218-2224. doi:10.1016/j.bios.2010.02.023.

[17] L. Gao, J. Wang, X. Li, Y. Yan, C. Li, J. Pan, A core-shell surface magnetic molecularly imprinted polymers with fluorescence for -cyhalothrin selective recognition, Anal. Bioanal. Chem. 406 (2014) 7213-7220. doi:10.1007/s00216-0148126-8.

[18] M. Niu, C. Pham-Huy, H. He, Core-shell nanoparticles coated with molecularly imprinted polymers: a review, Microchim. Acta. 183 (2016) 2677-2695. doi:10.1007/s00604-016-1930-4.

[19] Y.K. Lv, C.X. Zhao, P. Li, Y.D. He, Z.R. Yang, H.W. Sun, Preparation of doxycycline-imprinted magnetic microspheres by inverse-emulsion suspension polymerization for magnetic dispersion extraction of tetracyclines from milk samples, J. Sep. Sci. 36 (2013) 2656-2663. doi:10.1002/jssc.201300429.

[20] L. Chen, B. Li, C. Nie, J. Kong, X. Liu, T. Wen, L. Yuan, P. Dai, Y. Lin, X. Zhou, S. Mei, S. Thang, Application of magnetic molecularly imprinted polymers in analytical chemistry, Anal. Methods. 4 (2012) 2613. doi:10.1039/c2ay25354b.

[21] T. Kamra, T. Zhou, L. Montelius, J. Schnadt, L. Ye, Implementation of molecularly imprinted polymer beads for surface enhanced raman detection, Anal. Chem. 87 (2015) 5056-5061. doi:10.1021/acs.analchem.5b00774.

[22] J.L. Chen, Determination of tetracycline using imprinted polymethacrylates along with fluorescent CdTe quantum dots on plastic substrates, Microchim. Acta. 184 (2017) 1335-1343. doi:10.1007/s00604-017-2118-2. 
[23] J. Hou, H. Li, L. Wang, P. Zhang, T. Zhou, H. Ding, L. Ding, Rapid microwaveassisted synthesis of molecularly imprinted polymers on carbon quantum dots for fluorescent sensing of tetracycline in milk, Talanta. 146 (2016) 34-40. doi:10.1016/j.talanta.2015.08.024.

[24] S. Han, X. Li, Y. Wang, C. Su, A core-shell Fe3O4 nanoparticle-CdTe quantum dotmolecularly imprinted polymer composite for recognition and separation of 4nonylphenol, Anal. Methods. 6 (2014) 2855. doi:10.1039/c3ay41924j.

[25] B. Li, Z. Zhang, J. Qi, N. Zhou, S. Qin, J. Choo, L. Chen, Quantum Dot-Based Molecularly Imprinted Polymers on Three- Dimensional Origami Paper Microfluidic Chip for Fluorescence Detection of Phycocyanin, ACS Sensors. 2 (2017) 243-250. doi:10.1021/acssensors.6b00664.

[26] E. Oh, R. Liu, A. Nel, K.B. Gemill, M. Bilal, Y. Cohen, I.L. Medintz, Meta-analysis of cellular toxicity for cadmium-containing quantum dots, Nat Nano. 11 (2016) 479-486. http://dx.doi.org/10.1038/nnano.2015.338.

[27] T. Chen, J. Zhao, Z. Guo, B. Zhang, Grafting of doxycycline imprinted polymers on silica surface for selective solid-phase extraction in beef samples, Anal. Methods. 4 (2012) 4237. doi:10.1039/c2ay26084k.

[28] J. Tashkhourian, G. Absalan, M. Jafari, S. Zare, A rapid and sensitive assay for determination of doxycycline using thioglycolic acid-capped cadmium telluride quantum dots, Spectrochim. Acta Part A Mol. Biomol. Spectrosc. 152 (2016) 119-125. doi:10.1016/j.saa.2015.07.063.

[29] B. Gürler, S.P. Özkorucuklu, E. Kir, Voltammetric behavior and determination of doxycycline in pharmaceuticals at molecularly imprinted and non-imprinted 
overoxidized polypyrrole electrodes, J. Pharm. Biomed. Anal. 84 (2013) 263-268. doi:10.1016/j.jpba.2013.06.009.

[30] J.L. Rufino, F.C.B. Fernandes, M.S. Ruy, H.R. Pezza, L. Pezza, A simple spectrophotometric method for the determination of tetracycline and doxycycline in pharmaceutical formulations using chloramine-T, Eclética Química. 35 (2010) 139146. doi:10.1590/S0100-46702010000400018. 


\section{Figure Captions}

Fig. 1. Overview of core-shell molecular imprinting of the MIP composites.

Fig. 2. TEM images of (A) $\mathrm{FeO}_{\mathrm{x}}$, (B) $\mathrm{FeO}_{\mathrm{x}} @ \mathrm{SiO}_{2}$, (C) $\mathrm{FeO}_{\mathrm{x}} @ \mathrm{SiO}_{2}-\mathrm{FMIPs}$ and corresponding $\mathrm{FeO}_{\mathrm{x}} @ \mathrm{SiO}_{2}$-FNIPs.

Fig. 3. IR spectra of $\mathrm{FeO}_{\mathrm{x}}, \mathrm{FeO}_{\mathrm{x}} @ \mathrm{SiO}_{2}$ and $\mathrm{FeO}_{\mathrm{x}} @ \mathrm{SiO}_{2}-\mathrm{FMIPs}$ and corresponding $\mathrm{FeO}_{\mathbf{x}} @ \mathrm{SiO}_{2}$-FNIPs .

Fig. 4. Fluorescence image of $\mathrm{FeO}_{\mathrm{x}} @ \mathrm{SiO}_{2}-\mathrm{FMIPs}$.

Fig. 5. Fluorescence emission quenching spectra of (A) $\mathrm{FeO}_{\mathrm{x}} @ \mathrm{SiO}_{2}-\mathrm{FMIPs}$ and (B) $\mathrm{FeO}_{\mathrm{x}} @ \mathrm{SiO}_{2}$-FNIPs in presence of different concentrations of doxycycline from 0 to 6 $\boldsymbol{\mu M}$.

Fig. 6. (A) Linear and (B) non-linear Stern-Volmer plots showing the doxycycline concentration-dependent quenching of $\mathrm{FeO}_{\mathrm{x}} @ \mathrm{SiO}_{2}$-FMIPs; (C) linear and (D) nonlinear Stern-Volmer plots showing the quenching of the corresponding $\mathrm{FeO}_{\mathbf{x}} @ \mathrm{SiO}_{2}$ FNIPs.

Fig. 7. Selectivity of $\mathrm{FeO}_{\mathrm{x}} @ \mathrm{SiO}_{2}-\mathrm{FMIPs}$ and corresponding $\mathrm{FeO}_{\mathrm{x}} @ \mathrm{SiO}_{2}-\mathrm{FNIPs}$ towards common antibiotic residues.

Table Captions

Table 1. Percentage recoveries and RSD of doxycycline from spiked pork plasma samples $(\mathbf{n}=3)$. 